Address for Correspondence: Prof. Emine Yilmaz Sipahi Department of Medical Pharmacology, Bulent Ecevit University,

Faculty of Medicine, Zonguldak,

Turkey.

E-mail: dresipahi@yahoo.com

\begin{tabular}{|l|}
\hline Access this article online \\
Website: \\
www.intern-med.com \\
DOI: \\
10.4103/2224-4018.147738 \\
Quick Response Code: \\
\hline \\
\hline
\end{tabular}

\title{
Experimental models of acute respiratory distress syndrome
}

\author{
Emine Yilmaz Sipahi \\ Department of Medical Pharmacology, Bulent Ecevit University, Faculty of Medicine, Zonguldak, Turkey
}

\section{ABSTRACT}

Acute respiratory distress syndrome (ARDS) is a major cause of morbidity, death and cost in intensive care units. ARDS was first described in 1967, and the new definition was determined as the Berlin definition in 2011 in Berlin. ARDS is a syndrome of inflammation and increased permeability of the blood-gas barrier. Despite intensive research, treatments remain limited and supportive therapies represent the mainstay of the treatment of ARDS. This inability of therapeutic modalities largely depends on the complex pathogenesis of this syndrome with multiple overlapping signaling pathways activated depending on the type of lung injury. Today, this syndrome is still associated with a high morbidity and mortality. Animal models provide us a bridge between bench and bedside. Numerous different models have been developed in order to establish the properties of ARDS, but, to date, no single animal model that mimics all of the characteristics of ARDS in humans has been developed, and most of the existing animal models are relevant only for limited aspects of human ARDS. Furthermore, each animal model has unique features that affect responses to treatment. Therefore, when choosing an animal model of ARDS, to take into account the key feature of ARDS as a working hypothesis to be tested and then create the most appropriate model to exhibit those features is important. The goal of this review is to summarize the properties of the most commonly used experimental animal models of ARDS after mentioning briefly the causes and pathophysiology.

Key words: Acute respiratory distress syndrome, experimental models, respiratory failure

\section{INTRODUCTION}

Acute respiratory distress syndrome (ARDS) is a syndrome with multiple risk factors that trigger the acute onset of respiratory insufficiency. Until recently, the most accepted definition of ARDS was the American-European Consensus Conference (AECC) definition published in 1994. ${ }^{[1]}$ According to this definition, ARDS was defined as: The acute onset of respiratory failure, bilateral infiltrates on chest radiography, hypoxemia as defined by a $\mathrm{PaO}_{2} / \mathrm{FiO}_{2}$ ratio $\leq 200 \mathrm{mmHg}$ and no evidence of left atrial hypertension or a pulmonary capillary pressure $<18 \mathrm{mmHg}$ (if measured) to rule out cardiogenic edema. Acute lung injury (ALI), the less severe form of acute respiratory failure, was different from ARDS only for the degree of hypoxemia; in fact, it was defined as 200 $<\mathrm{PaO}_{2} / \mathrm{FiO}_{2} \leq 300 \mathrm{mmHg}$.

\section{The Last Definition of ARDS:} The Berlin Definition (2011)

Over the past 18 years of practice, the diagnostic accuracy of the ARDS definition by the AECC has been questioned. Owing to the concerns about the specificity and reliability of this definition, the 1994 definition was revised in Berlin and hence the new definition was coined as the Berlin definition. ${ }^{[2]}$ The goal of developing the Berlin definition was to try and improve the feasibility, reliability, validity and objective evaluation of its performance.

In the Berlin definition, there is no use of the term "Acute Lung Injury," and it was abandoned. The committee felt that this term was used inappropriately in many contexts and hence was not helpful. On the other hand, ARDS is categorized as being mild, moderate or severe according to the value of the $\mathrm{PaO}_{2} / \mathrm{FiO}_{2}$ ratio [Table 1]. ${ }^{[3]}$ Importantly, the $\mathrm{PaO}_{2} / \mathrm{FiO}_{2}$ ratio value is 


\begin{tabular}{|c|c|}
\hline \multicolumn{2}{|c|}{ The Berlin definition of acute respiratory distress syndrome } \\
\hline Timing & $\begin{array}{l}\text { Within } 1 \text { week of a known clinical insult or } \\
\text { new or worsening respiratory symptoms }\end{array}$ \\
\hline Chest imaging & $\begin{array}{l}\text { Bilateral opacities - not fully explained by } \\
\text { effusions, lobar/lung collapse or nodules }\end{array}$ \\
\hline \multirow[t]{2}{*}{ Origin of edema } & $\begin{array}{l}\text { Respiratory failure not fully explained by } \\
\text { cardiac failure or fluid overload }\end{array}$ \\
\hline & $\begin{array}{l}\text { Need objective assessment (e.g., } \\
\text { echocardiography) to exclude hydrostatic } \\
\text { edema if no risk factor present }\end{array}$ \\
\hline \multicolumn{2}{|l|}{ Oxygenation } \\
\hline Mild & $\begin{array}{l}200 \mathrm{mmHg}<\mathrm{PaO}_{2} / \mathrm{FiO}_{2} \leq 300 \mathrm{mmHg} \text { with } \\
\text { PEEP or CPAP } \geq 5 \mathrm{cmH}_{2} \mathrm{O}\end{array}$ \\
\hline Moderate & $\begin{array}{l}100 \mathrm{mmHg}<\mathrm{PaO}_{2} / \mathrm{FiO}_{2} \leq 200 \mathrm{mmHg} \text { with } \\
\text { PEEP } \geq 5 \mathrm{cmH}_{2} \mathrm{O}\end{array}$ \\
\hline Severe & $\begin{array}{l}\mathrm{PaO}_{2} / \mathrm{FiO}_{2} \leq 100 \mathrm{mmHg} \text { with PEEP } \geq 5 \\
\mathrm{cmH}_{2} \mathrm{O}\end{array}$ \\
\hline
\end{tabular}

CPAP: Continuous positive airway pressure, $\mathrm{FiO}_{2}$ : Fraction of inspired oxygen, $\mathrm{PaO}_{2}$ : Partial pressure of arterial oxygen, PEEP: Positive endexpiratory pressure

considered only with a CPAP or PEEP value of at least $5 \mathrm{cmH}_{2} \mathrm{O}$.

The timing of acute onset of respiratory failure to make a diagnosis of ARDS is clearly defined in the Berlin definition. It defines the exposure to a known risk factor or worsening of the respiratory symptoms within 1 week.

ARDS is still among the most fatal diseases with a high mortality rate in intensive care units. In the Berlin definition clinical study cohort, the mortality rate was $27 \%$ for mild, $32 \%$ for moderate and $45 \%$ for severe ARDS. ${ }^{[2]}$ Although worsening oxygenation is a risk factor for ARDS mortality, patients generally die from multisystem organ failure or progressive underlying illness; only a minority of ARDS patients (13-19\%) die from refractory respiratory failure. ${ }^{[3-5]}$ In other words, the degree of lung damage or extrapulmonary disease and various factors that can accompany the same also play a role in mortality rate.

Epidemiological studies have shown that in most cases a few common reasons may be effective alone or in combination. The most common causes of ARDS are sepsis, pneumonia, aspiration of gastric contents, trauma and emergency transfusions. These causative factors that precipitate ARDS can be grouped broadly into direct and indirect factors. ${ }^{[6]}$ Direct factors include bacterial and viral infections in the lung, aspiration of gastric contents, inhalational injury, pulmonary contusion, pulmonary vasculitis, drowning, etc.; all of these factors cause direct injury to the airway and alveolar epithelium and other structures in the airspaces. Indirect factors include systemic infections that cause the sepsis syndrome, major trauma, pancreatitis, severe burn, blood transfusions (transfusion associated acute lung injury-TRALI), effects of systemic medications, illicit drugs, etc. ${ }^{[3,6,7]}$

ARDS is a syndrome of acute respiratory failure, secondary to increased permeability and non-cardiogenic pulmonary edema. ${ }^{[8]}$ Alveolar edema accumulates in ARDS mainly because of the increase in permeability of the capillary (endothelium)-alveolar (epithelium) barrier. The American-European consensus conference defined ARDS as a "syndrome of inflammation and increased permeability." "[7] Thus, it is now widely accepted that the pathophysiology of ARDS is driven by an aggressive inflammatory reaction that damages the alveolar-capillary unit. ${ }^{[7-9]}$ However, the mechanism(s) of lung injury, time course, inflammatory pathway(s) and cell repair process(es) are not well understood.

The pathology of ARDS can be divided into three overlapping stages, including exudative, proliferative and fibrotic. ${ }^{[4,10]}$ The causative factors that precipitate ARDS initiate the exudative phase. In this acute inflammatory stage, release of proinflammatory cytokines, influx of neutrophils and impaired endothelial cell barrier function occur. Accumulation of protein-rich fluid in distal airspaces and decreased surfactant production by type II epithelial cells leads to respiratory failure. [4,9,10] These early events are followed by the proliferative phase, which develops 2-7 days after beginning of lung injury. This phase is characterized by the proliferation of type II pneumocytes, early fibrotic changes and myointimal thickening of the alveolar capillaries. In some individuals, the proliferative phase progresses to a fibrotic stage that is associated with increased collagen deposition, a prolonged period of ventilation-perfusion mismatching and diminished compliance of the lung. This pathophysiological process results in severe respiratory distress. ${ }^{[4,9,10]}$ There has been intense investigation into the pathophysiologic events relevant to the earliest stages of disease and much has been learnt, particularly in animal models, about the changes in endothelial and epithelial homeostasis in the lung in the first $24-48 \mathrm{~h}$ after initial lung injury. ${ }^{[1]}$ Intervention based on interruption of pathways identified in the acute phase has been effective when provided as pre-treatment in animal models, but the requirement for early delivery, coupled with the likely pathophysiologic complexity of human ARDS, has markedly limited clinical utility. ${ }^{[0]}$

Complicated processes that develop in acute lung injury begin following a triggering event after some cellular and chemical mediators become activated. These mediators cause free oxygen products and toxic enzymes to emerge for tissues that are released from cells, especially from neutrophils. In the end, a sequence of events leading to 
alveolar and capillary damage begins. Various biological processes such as inflammation, apoptosis and thrombosis take active roles in the pathogenesis of ARDS..$^{[5,11,12]}$

Numerous cellular and biochemical mediators play a role in the pathogenesis of ARDS. Cytokines, which are activated by stimulated complement-mediated neutrophils, play a major role. Proinflammatory cytokines such as tumor necrosis factor-alfa, interleukin-1, interleukin-6 and interleukin- 8 are secreted as a response to various stimulants. ${ }^{[5,7,8,10,12-15]}$ Proinflammatory cytokines can be generated from inflammatory cells, fibroblasts and epithelial cells of the lungs. Afterwards, substantial neutrophil accumulation takes place in the lungs and these cells become activated and secrete toxic mediators such as reactive oxygen derivatives and protease that lead to capillary endothelial and alveolar epithelial damage. Like neutrophils, proteolytic enzymes and reactive oxygen derivatives are secreted from alveolar and interstitial macrophages in the lung tissue, which causes tissue damage. Activated arachidonic acid metabolites (platelet activating factor), tromboxane-A2 (TXA2) and leukotriene-B4 (LTB4) cause increased permeability and cellular damage. Nitric oxide (NO), which is generated in many cells, especially in sepsis, is a strong endogenous vasodilator; with increased permeability and reactive oxygen species, it plays an active role in alveolar and capillary damage. [, $, 7,8,10,12-15]^{2}$ None of the processes that have been identified to date are entirely responsible for the development of ARDS alone, and it involves complicated relationships that cannot still be exactly explained.

Although mortality has declined since two decades ago, initial progress in reducing ARDS mortality is likely due to increased implementation of a low tidal-volume mechanical ventilation strategy that reduces further lung injury, systemic inflammation and subsequent multisystem organ failure. However, among patients who receive low tidal-volume ventilation, mortality rates remain unchanged. On the other hand, pharmacotherapies have a very limited role in the management of ARDS and additional treatments for ARDS are sorely needed. ${ }^{[3,4,8,16]}$ Therapeutic strategies for ARDS focus upon treating the underlying etiology and providing supportive care that reduces the progression of lung injury. At this point, animal experiments are an indispensable necessity for improving treatment and reducing mortality rate.

Animal models provide a bridge between patients and the laboratory bench. However, to date, no single animal model that reproduces all of the characteristics of ARDS in humans has been developed, and most of the existing animal models are relevant only for limited aspects of human ARDS. ${ }^{[17]}$ Because of the complexity of ARDS pathogenesis in humans, each model of lung injury in animals has its own limitations. Thus, the choice of a particular model should be made only after the specific features of each have been considered.

The human ARDS definition is not practical in animal studies, particularly in small animals. Therefore, in order to define ARDS, using histopathological criteria similar to those seen in humans would be a more accurate approach. In humans, the pathological correlate of ARDS is diffuse alveolar damage characterized by inflammatory infiltrates, thickened alveolar septae and deposition of hyaline membranes. Although none of the available animal models of ARDS reproduce all of the pathologic features of diffuse alveolar damage and the criteria used to define ARDS in humans, lacking one of these factors does not mean that this model is not a form of ARDS. ${ }^{[17]}$ Consequently, when choosing an animal model of ARDS, the key feature to be tested should be determined and then the most appropriate model to exhibit those features should be created. If you want to examine the passage of neutrophils into the lung, you can choose the lipopolysaccharide (LPS) model characterized by alveolar neutrophilia or, if are you interested in epithelial damage, you can choose the lung ischemia/reperfusion model. ${ }^{[18]}$

The ideal animal model of ARDS should imitate one or more features of human ARDS, including physiological and pathological changes. These changes can be summarized as detection of rapid development process measured in hours, gas exchange abnormalities, decreased pulmonary compliance, injury of the lung parenchyma (endothelium, interstitium, epithelium) and increased permeability in the alveolar-capillary membrane. ${ }^{[6,7,17-19]}$ As noted above, we have no a certain model that provides all these features. Therefore, the most important question is how many features are necessary in order to identify it as ARDS. It is hard to answer this question and there is no one right answer. Therefore, when choosing an ARDS animal model, it is important to consider the ARDS key feature that is to be studied with a study hypothesis and then select a model that can exhibit those features.

\section{Limitations of ARDS models}

Most of the animal models of ARDS mimic the clinical disorders associated with ARDS in humans, but there are certain limitations of animal models. The formation of pathology takes hours or days in humans, ${ }^{[7]}$ whereas the monitored period is shorter in animal models. We observed lighter pathology than in humans and most animal models do not include ventilation and fluid management interventions that may be crucial factors in determining outcome in humans (these applications should be added to animal experiments if possible). In this case, monitoring 
difficulties are experienced. The species and the size of the animal may cause differences in application. Larger animals that more closely replicate human disease, such as primates, are expensive and require specialized research facilities. Smaller animals, such as mice, are much more widely accessible to researchers and are a very powerful research tool as they can be genetically manipulated in multiple ways to facilitate the detailed mechanistic study of complex pathways. ${ }^{[20]}$ Therapeutic agents in experimental studies are given before the onset of ARDS, whereas the clinical diagnosis and treatment of ARDS is delayed. Animal experiments are performed on young animals; however, human patients are mostly elderly and may have many medical problems such as diabetes, coronary artery disease or kidney or liver failure. Biochemical markers (parameters, tests) measured in bronchoalveloar lavage fluid (BAL), plasma and edema fluid may not be correlated with their biological activities (markers may not be correlated with clinical diagnosis and pathogenesis, while these factors take part in extracellular transmission, they may not be released extracellularly and may not be measured). ${ }^{[6,17-20]}$ Despite all these limitations, the complex pathogenesis of ARDS makes animal models a necessity in the study of this disorder. Matuta-Bello et al. discussed each model in great detail with its advantages, disadvantages and methodologies in their review (19). Another important issue related to animal experiments is these models could and should focus on both systemic (e.g., renal failure, hepatic and hematologic dysfunction) and pulmonary injury and may also provide insights about the interactions between them. ${ }^{[7]}$

Different animal models have been developed in order to imitate human ARDS pathology. It is possible to divide these models into two groups as direct or indirect lung injury models. ${ }^{[6,17-20]}$ Direct models include administration of intratracheal or intranasal bacteria or bacterial product such as lipopolysaccaride (LPS), administration of acid such as hydrochloric acid $(\mathrm{HCl})$ or gastric particle to create an acid aspiration, administration of high inspired fraction of oxygen, surfactant depletion via serial lavages with $0.9 \%$ sodium chloride $(\mathrm{NaCl})$, forming ischemia/ reperfusion by clamping hilus or exposure to mechanical stress via mechanical ventilation at high tidal volumes. ${ }^{[18]}$ The indirect model group generally includes models based on the formation of sepsis. Some of them are cecal ligation and puncture, ${ }^{[21]}$ intravenous bacteria or LPS administration ${ }^{[2]}$ and mesenteric ischemia/reperfusion models. ${ }^{[21,22]}$ This category also includes an oleic acid model reproduces patients with multiple bone fractures. ${ }^{[18]}$ Intraperitoneal application of ANTU ( $\alpha$-naphthylthiourea) is also an appropriate approach for the indirect lung injury model characterized by acute pulmonary vascular injury in animals. ${ }^{[11,23-25]}$ In order to create a similar human ARDS model, various injury models can be combined (i.e., cecal ligation and puncture followed by hemorrhage or saline lavage followed by mechanical ventilation or intraperitoneal LPS injection followed by intravenous oleic acid). ${ }^{[18,26]}$

\section{Experimental ARDS models}

In humans, pneumonia and sepsis are the two most common predisposing conditions for the development of ARDS. ${ }^{[20,27]}$ Inhalation of aerosolized bacteria or direct intranasal, endotracheal or endobronchial instillation is used as an experimental model of pneumonia in animals. Tissue injury in this model is characterized by accumulation of neutrophils in the alveolar and interstitial spaces and alveolar wall thickening and hyaline membrane formation. ${ }^{[17]}$

In order to create sepsis, three applications are performed: ${ }^{[1,28,29]}(1)$ injection of live bacteria (bacteria can be administered intravenously as a bolus or as a continuous infusion, or into the peritonium), (2) development of endogenous infection/alteration of the endogenous protective barrier of the animal (e.g., cecal ligation/ puncture [CLP]) and (3) injection of bacterial structures (e.g., LPS, Gram negative bacterial endotoxin). Dejager et al. summarized the advantages and disadvantages of the most commonly used experimental models for sepsis. ${ }^{[2]}$

Live bacterial applications generate a severe infection leading to a secondary sepsis syndrome. ${ }^{[30]}$ In this model, initial hypotension and leukopenia are followed by septic shock, intravascular coagulation and death if the inoculum is high. ${ }^{[19]}$ If the animals survive, polymorphonuclear leukocytes (PMN) sequestration in the lungs, increase in pulmonary microvascular permeability, interstitial edema, increase in pulmonary arterial pressure and intravascular thrombosis occur. ${ }^{[19]}$

CLP is one of the most used sepsis model. Compared with other models, CLP provides a better representation of the complexity of human sepsis ${ }^{[21,29-31]}$ and is considered by many investigators to be the crucial pre-clinical test for any treatment of human sepsis. ${ }^{[29,32]}$ Dejager et al. summarize the advantages and the reasons of popularity of this method, including the pathogens originating from within the host (mimic traumatic injury leading to peritonitis in humans), a simple procedure, polymicrobial nature with a localized infectious focus and bacterial products released in the periphery that lead to septicemia. ${ }^{[29]}$ A relatively new model like CLP is colon ascendens stent peritonitis (CASP). In this model, a stent of defined diameter is surgically inserted into the ascending colon, and this causes continuous influx of enteric bacteria into the peritoneal cavity. ${ }^{[2,31,32]}$ This model is not well characterized yet and has more difficult surgical procedure. Berguer et al. described a technique of laparoscopic cecal ligation and puncture in the rat that 
may facilitate the study of minimally invasive surgery and peritonitis. ${ }^{[33]}$

LPS is a glycolipid and is located on the outer membrane of Gram negative bacteria. A large part of the biological effects of LPS result from lipid A, which is a part of its content, and LPS has a purified glycolipid structure. LPS is an important mediator for Gram negative bacterial sepsis. Administration of systemic LPS is one of the first approaches to form a bacterial sepsis model. ${ }^{[7,19,21]}$ Sepsis in humans can be modeled in mice by using the Gram negative bacterial endotoxin LPS, which can be administered either directly to the lungs through intratracheal injection or inhalation or given intraperitoneally or intravenously to incite a systemic inflammatory response. Intratracheal application of LPS leads to acute inflammatory cell influx to the lung. Intraperitoneal LPS activates systemic inflammation and is associated with a mild lung injury. This injury can be augmented with either repeated injections of LPS or the implantation of an LPS pump in the peritoneal cavity to continually release LPS for hours, or even days. ${ }^{[2,28]}$ In general, administration of LPS does not cause the severe endothelial and epithelial injury that occurs in humans with ARDS. ${ }^{[19,34]}$

Another commonly used model of lung injury is mechanical ventilation. In this model, mechanical ventilation may cause pulmonary inflammation and lung injury in animals (ventilator-induced lung injury, VILI), which correlates with human ventilator-induced lung injury. ${ }^{[17,19,20]}$ This model is the only model affected by clinical application performed in humans. The main advantage of this model is that it is clinically relevant and provides opportunities to make changes in clinical practice, and the main disadvantage is the complexity of the model. ${ }^{[19]}$ In this model, in order to get more severe injury, higher tidal volumes should be used. ${ }^{[17,20,35]}$

Aspiration of gastric contents is an important risk factor for ARDS. Acid-induced lung injury models try to mimic the clinical situation of gastric juice aspiration. Generally, this model involves instillation of $1-4 \mathrm{~mL} / \mathrm{kg}$ of $\mathrm{HCl}$ with a $\mathrm{pH}$ between 1 and 2 into the airways of experimental animals. ${ }^{[3]}$ Ischemia followed by reperfusion, either in the lungs or in distant vascular beds, can lead to lung injury. A pulmonary ischemia reperfusion model can be performed by clamping of the pulmonary artery or hilum. ${ }^{\left[{ }^{37]}\right.}$ Ischemia reperfusion of the non-pulmonary artery model can be performed by clamping of the superior mesenteric artery or supraceliac aorta (hindlimb ischemia) or without surgery by applying tourniquets to the extremities. ${ }^{[19]}$ Therefore, two general models including "pulmonary" and "nonpulmonary" ischemia reperfusion injuries have been defined to simulate ARDS.
Oleic acid is another model of lung injury. This model is improved to imitate lipid emboli. Lipid emboli is mostly observed as a result of long bone fractures or major traumas, and, after intravenous administration, endothelium damage starts within $1 \mathrm{~min} \cdot{ }^{[19]}$ Oleic acid directly causes endothelium toxicity. ${ }^{[3]}$ Another commonly used model of lung injury is hyperoxia, where mice breathe a high partial pressure of oxygen that is highly toxic to the alveolar epithelium and causes extensive alveolar epithelial injury with only a modest amount of inflammation. However, the direct relationship to human ARDS is unclear with this model. ${ }^{[20,39]}$

Depletion of surfactant with saline lavage is another model of ARDS. Repeated saline lavage $(0.9 \% \mathrm{NaCl})$ decreases surfactant lipid concentration in alveolar surface fluid and changes alveolar surface tension. Depletion of surfactant leads to alveolar collapse and disruption of alveolar host defense. ${ }^{[1,40]}$ Intratracheal, intravenous, intraperitoneal or subcutaneous administration of antineoplastic antibiotic drug bleomycin is a preferred way to mimic pulmonary fibrosis. Bleomycin leads to production of oxygen radicals, DNA breaks and, finally, cell death. ${ }^{\left[{ }^{19]}\right.}$ ANTU is a chemical agent developed as rodenticide. ${ }^{[21,23-25]}$ It has been determined that it develops dose- and time-dependent acute lung injury. Morphological studies performed in electron and light microscopy have shown that the target structure in lung injury mechanisms of ANTU is capillary endothelium cells. ${ }^{[41-47]}$ In a standard experimental model, when the animals (mostly rats and mice) are administered ANTU intraperitoneally, within $4 \mathrm{~h}$, pulmonary edema and pleural effusion develop reaching a maximum level.

\section{CONCLUSION}

ARDS still represents a deadly form of respiratory failure and pharmacotherapy has not passed the experimental stage and has a very limited role in the management of ARDS. At this point, development of experimental models and therapies can provide desirable reduction in the mortality rates. The main problem about ARDS treatment is the complex pathogenesis of this syndrome. Animal models provide a bridge between patients and the laboratory bench. Although no single animal model that reproduces all of the characteristics of ARDS in humans has been developed, improvement of the treatment about specific features of models will make progress in the treatment of ARDS.

\section{REFERENCES}

1. Bernard GR, Artigas A, Brigham KL, Carlet J, Falke K, Hudson L. et al . The American-European Consensus Conference on ARDS. Definitions, mechanisms, relevant outcomes, and clinical trial coordination. Am J Respir Crit Care Med 1994;149:818-24. 
2. ARDSDefinition Task Force, Ranieri VM, Rubenfeld GD, Thompson BT, Ferguson ND, Caldwell E, Fan E, et al. Acute Respiratory Distress Syndrome: The Berlin Definition. JAMA 2012;307:2526-33

3. Fanelli V, Vlachou A, Ghannadian S, Simonetti U, Slutsky AS, Zhang H. Acute respiratory distress syndrome: New definition, current and future therapeutic options. J Thorac Dis 2013;5:326-34.

4. Walkey AJ, Summer R, Ho V, Alkana P. Acute respiratory distress syndrome: Epidemiology and management approaches. Clin Epidemiol 2012;4:159-69.

5. Villar J, Blanco J, Añón JM, Santos-Bouza A, Blanch L, Ambros A, et al. The ALIEN Network: İncidence and outcome of acute respiratory distress syndrome in the era of lung protective ventilation. Intensive Care Med 2011;37:1932-41.

6. Martin TR, Matute-Bello G. Experimental models and emerging hypotheses for acute lung injury. Crit Care Clin 2011;27:735-52.

7. Matthay MA, Zimmerman GA, Esmon C, Bhattacharya J, Coller B, Doerschuk $\mathrm{CM}$, et al. Future research directions in acute lung injury: Summary of a National Heart, Lung, and Blood Institute Working Group. Am J Respir Crit Care Med 2003;167:1027-35.

8. Dushianthan A, Grocott MP, Postle AD, Cusack R. Acute respiratory distress syndrome and acute lung injury. Postgrad Med J 2011;87:612-22.

9. Tsushima K, King LS, Aggarwal NR, De Gorordo A, D'Alessio FR, Kubo K. Acute lung injury review. Intern Med 2009;48:621-30.

10. Bellingan GJ. The pulmonary physician in critical care * 6 : The pathogenesis of ALI/ARDS. Thorax 2002;57:540-6.

11. Ware LB, Matthay MA. The acute respiratory distress syndrome. N Engl J Med 2000;342:1334-49.

12. Boyle AJ, McNamee JJ, McAuley DF. Biological therapies in the acute respiratory distress syndrome. Expert Opin Biol Ther 2014;14:969-81.

13. Puneet $\mathrm{P}$, Moochhala $\mathrm{S}$, Bhatia $\mathrm{M}$. Chemokines in acute respiratory distress syndrome. Am J Physiol Lung Cell Mol Physiol 2005;288:L3-15.

14. Tasaka S, Hasegawa N, Ishizaka A. Pharmacology of acute lung injury. Pulm Pharmacol Ther 2002;15:83-95.

15. Varisco BM. The pharmacology of acute lung injury in sepsis. Adv Pharmacol Sci 2011;2011:254619.

16. Duggal A, Ganapathy A, Mohana Ratnapalan M, Adhikari NK. Pharmacological treatments for acute respiratory distress syndrome: Systematic review. Minerva Anestesiol 2014 Jun 17 [Epud ahead of print].

17. Mutate-Bello G, Downey G, Moore BB, Groshong SD, Matthay MA, Slutsky AS, et al. on behalf of the Acute Lung Injury in Animals Study Group. An official American Thoracic Society Workshop Report: Features and measurements of experimental acute lung injury in animals. Am J Respir Cell Mol Biol 2011;44:725-38.

18. Matute-Bello G, Matthay MA. Animal models of acute lung injury. Available from: http://www.thoracic.org/clinical/critical-care/criticalcare-research/animal-models-of-acute-lung-injury.php, 2013 [Last accessed on 2014].

19. Mutate-Bello G, Frevert CW, Martin TR. Animal models of acute lung injury. Am J Physiol Lung Cell Mol Physiol 2008;295:L379-99.

20. Bastarache JA, Blackwell TS. Development of animal models for the acute respiratory distress syndrome. Dis Model Mech $2009 ; 2: 218-23$

21. Kaynar G, Yurdakan G, Comert F, Yilmaz-Sipahi E. Effects of peripheral benzodiazepine receptor ligand Ro5-4864 in four animal models of acute lung injury. J Surg Res 2013;182:277-84.

22. Tekin IO, Sipahi EY, Comert M, Acikgoz S, Yurdakan G. Low-density lipoproteins oxidized after intestinal ischemia/reperfusion in rats. J Surg Res 2009;157:e47-54.

23. Cunningham AL, Hurley JV. Alpha-naphthyl-thiourea-induced pulmonary oedema in the rat: A topographical and electronmicroscope study. J Pathol 1972;106:25-35.

24. Erdem MK, Yurdakan G, Yilmaz-Sipahi E. The effects of ketamine, midazolam and ketamine/xylazine on acute lung injury induced by alpha-naphthylthiourea (ANTU) in rats. Adv Clin Exp Med 2014;23:343-51.

25. Atalay F, Yurdakan G, Yilmaz-Sipahi E. The Effect of the endothelin receptor antagonist tezosentan on acute lung injury induced by alphanaphthylthiourea in rats. Kaohsiung J Med Sci 2012;28:72-8.

26. Voelker M, Fichtner F, Kasper M, Kamprad M, Sack U, Kaisers U, et al. Characterization of a double-hit murine model of Acute Respiratory Distress Syndrome. Clin Exp Pharmacol Physiol 2014;41:844-53.
27. Varisco BM. The pharmacology of acute lung injury in sepsis. Adv Pharmacol Sci 2011;2011:254619.

28. Everhart MB, Han W, Sherrill TP, Arutiunov M, Polosukhin VV, Burke JR, et al. Duration and intensity of NF-kappaB activity determine the severity of endotoxin-induced acute lung injury. J Immunol 2006;176:4995-5005.

29. Dejager L, Pinheiro I, Dejonckheere E, Libert C. Cecal ligation and puncture: The gold standard model for polymicrobial sepsis? Trends Microbiol 2011;19:198-208.

30. Antonelli M, Azoulay E, Bonten M, Chastre J, Citerio G, Conti G, et al . Year in review in Intensive Care Medicine, 2008: II. Experimental, acute respiratory failure and ARDS, mechanical ventilation and endotracheal intubation. Intensive Care Med 2009;35:215-31.

31. Hubbard WJ, Choudhry M, Schwacha MG, Kerby JD, Rue LW $3^{\text {rd }}$, Bland KI, et al. Cecal ligation and puncture. Shock 2005;24 Suppl 1:52-7.

32. Maier S, Traeger T, Entleutner M, Westerholt A, Kleist B, Hüser N, et al. Cecal ligation and puncture versus colon ascendens stent peritonitis: Two distinct animal models for polymicrobial sepsis. Shock 2004;21:505-11.

33. Berguer R, Alarcon A, Feng S, Gutt C. Laparoscopic cecal ligation and puncture in the rat. Surg Endosc 1997;11:1206-8.

34. Wiener-Kronish JP, Albertine KH, Matthay MA. Differential responses of the endothelial barriers of the lung in sheep to Escherichia coli endotoxin. J Clin Invest 1991;88:864-75.

35. Lai CC, Liu WL, Chen CM. Glutamine attenuates acute lung injury caused by acid aspiration. Nutrients 2014;6:3101-16.

36. Reiss LK, Uhlig U, Uhlig S. Models and mechanisms of acute lung injury caused by direct insults. Eur J Cell Biol 2012;91:590-601.

37. Fard N, Saffari A, Emami G, Hofer S, Kauczor HU, Mehrabi A. Acute respiratory distress syndrome induction by pulmonary ischemiareperfusion injury in large animal models. J Surg Res 2014;189:274-84.

38. Lee SM, Choi H, Yang G, Park KC, Jeong S, Hong S. MicroRNAs mediate oleic acid-induced acute lung injury in rats using an alternative injury mechanism. Mol Med Rep 2014;10:292-300.

39. Lingappan K, Jiang W, Wang L, Couroucli XI, Barrios R, Moorthy B. Sex-specific differences in hyperoxic lung injury in mice: Implications for acute and chronic lung disease in humans. Toxicol Appl Pharmacol 2013;272:281-90.

40. Muellenbach RM, Kredel M, Zollhoefer B, Johannes A, Kuestermann J, Schuster $\mathrm{F}$, et al. Acute respiratory distress induced by repeated saline lavage provides stable experimental conditions for 24 hours in pigs. Exp Lung Res 2009;35:222-33.

41. Sipahi E, Hodoglugil U, Ercan ZS, Turker RK. Acute effect of endothelin-1 on lung oedema induced by alphanaphthylthiourea (ANTU). Pharmacol Res 1996;33:375-8.

42. Sipahi E, Hodoglugil U, Ustun H, Zengil H, Turker RK, Ercan ZS. An unexpected interaction between NG-nitro-Larginine methyl ester and L-arginine in alphanaphthylthiourea-induced pulmonary oedema in rats. Eur J Pharmacol 1997;321:45-51.

43. Sipahi EY, Ozel Tekin I, Comert M, Barut F, Ustun H, Sipahi TH. Oxidized low-density lipoproteins accumulate in rat lung after experimental lung edema induced by alphanaphthylthiourea (ANTU). Pharmacol Res 2004;50:585-91.

44. Comert M, Sipahi EY, Ustun H, Isikdemir F, Numanoglu G, Barut F, et al. Morphine modulates inducible nitric oxide synthase expression and reduces pulmonary oedema induced by alpha-naphthylthiourea. Eur J Pharmacol 2005;511:183-9.

45. Sipahi E, Ustun H, Niyazi Ayoglu F. Acute effects of thiopental, pentobarbital and urethane on lung oedema induced by alpha naphtylthiourea (ANTU). Pharmacol Res 2002;45:235-9.

46. Hanci V, Yurdakan G, Yurtlu S, Turan IO, Sipahi EY. Protective effect of dexmedetomidine in a rat model of a-naphthylthiourea-induced acute lung injury. J Surg Res 2012;178:424-30.

47. Richter CP. The physiology and cytology of pulmonary edema and pleural effusion produced in rats by alpha-naphthyl thiourea (ANTU). J Thorac Surg 1952;23:66-91.

How to cite this article: Sipahi EY. Experimental models of acute respiratory distress syndrome. J Transl Intern Med 2014;2:154-9.

Source of Support: Nil, Conflict of Interest: None declared. 